\title{
APPROXIMATE UNITARY EQUIVALENCE OF POWER PARTIAL ISOMETRIES
}

\author{
KENNETH R. DAVIDSON ${ }^{1}$
}

\begin{abstract}
Every power partial isometry (p.p.i.) in the Calkin algebra lifts to a p.p.i. in $B(H)$.
\end{abstract}

An element $u$ in a $C^{*}$ algebra is a power partial isometry (p.p.i.) if $\mathfrak{u}^{n}$ is a partial isometry for every integer $n \geq 1$. This notion was introduced by Halmos and Wallen [5], and they characterized all p.p.i.'s in $B(\not H)$. In a penetrating study [6], Herrero classifies p.p.i.'s up to their unitary orbits and up to unitary equivalence modulo the compacts. In this note, we show that every p.p.i. in the Calkin algebra lifts to a p.p.i. in $B(\mathcal{H})$. This is done by using a few straightforward computations involving the theory of $C^{*}$ extensions. Some of the results of [6] follow from this method as well.

In this paper, Hilbert spaces are always separable. The ideal of compact operators will be denoted by $K$. The canonical quotient map of $B(\not K)$ onto the Calkin algebra $B(\mathcal{H}) / K$ is denoted by $\pi$. Two elements $\mathfrak{s}$ and $t$ of the Calkin algebra are (strongly) compalent $(\mathfrak{s} \cong \mathfrak{t})$ if there is a unitary $U$ in $B(\mathcal{H})$ so that $t=\pi(U) \mathfrak{s} \pi\left(U^{*}\right)$. They are weakly compalent $\left(\mathfrak{s} \cong_{w} \mathfrak{t}\right)$ if there is a unitary $\mathfrak{u}$ in $B(\mathcal{H}) / \mathcal{K}$ so that $\mathbf{t}=\mathbf{u s u}^{*}$. For p.p.i.'s, these two notions may differ. Given a nuclear $C^{*}$ algebra $A$, Ext $A$ denotes the group of extensions of $K$ by $A$ modulo compalence $[1,3,8]$ and $\operatorname{Ext}_{w} \mathcal{A}$ denotes the quotient of $\operatorname{Ext} A$ modulo weak compalence. We use little more than the fact that when

$$
0 \rightarrow J \rightarrow A \rightarrow A / J \rightarrow 0
$$

is an exact sequence, and $\operatorname{Ext} J=0=\operatorname{Ext} A / J$, then $\operatorname{Ext} A=0$.

The first step is to classify p.p.i.'s up to algebraic equivalence by computing $C^{*}(T)$ for all p.p.i.'s. That is, given two p.p.i.'s $S$ and $T$, when is there a $C^{*}$ isomorphism $\phi$ of $C^{*}(S)$ onto $C^{*}(T)$ such that $\phi(S)=T$ ? For convenience, this will be written $S \sim T$. Let $N_{k}$ be the nilpotent Jordan cell of order $k$ acting on a $k$-dimensional Hilbert space, and let $S$ be the unilateral shift. If $\alpha$ belongs to $\mathbf{N}_{0} \cup\{\omega\}$, let $A^{(\alpha)}$ be the direct sum of $\alpha$ copies of $A$ acting on $\mathcal{H}^{(\alpha)}$, the direct sum of $\alpha$ copies of $\nvdash$. By [5], every p.p.i. $T$ represented on a Hilbert space can be decomposed as

$$
T \cong \bigoplus \sum_{k=1}^{\infty} N_{k}^{\left(\alpha_{k}\right)} \oplus S^{(\alpha)} \oplus S^{*(\beta)} \oplus V
$$

where $V$ is a unitary. (If $\alpha_{k}=0$, the summand is deemed to be absent.) The existence of the finite nilpotent blocks is easy to determine because there is a

Received by the editors July 9, 1983.

1980 Mathematics Subject Classification. Primary 47B99, 47C15; Secondary 46M20.

${ }^{1}$ Research partially supported by NSERC grant \#A3488. I would also like to thank the people at Indiana University for their hospitality during the period when this work was completed. 
polynomial $p_{k}(x, y)$ in two noncommuting variables such that $P_{k}=p_{k}\left(T, T^{*}\right)$ is a (selfadjoint) projection onto the summand $\mathcal{H}_{k}^{\left(\alpha_{k}\right)}$ on which $N_{k}^{\left(\alpha_{k}\right)}$ acts. The classification in [5] proceeds by pulling off all the nilpotent blocks, then one removes the isometric and coisometric parts, and what remains is the unitary.

This procedure is not possible in an arbitrary $C^{*}$ algebra because one can pull off only finitely many pieces. Also, if $T$ has no nilpotent blocks and is not unitary, then $C^{*}(T)$ has only a few possibilities up to algebraic equivalence. If $\Sigma$ is a nonempty compact subset of the unit circle $S^{1}$, let $V_{\Sigma}$ be a fixed unitary with spectrum $\Sigma$.

Proposition 1. (a) If $T$ is a p.p.i. with no nilpotent blocks, then there are only the following possibilities:

(a1) $T$ is unitary $\left(T T^{:}=I=T^{:} T\right)$, in which case the spectrum $\Sigma=\sigma(T)$ determines $C^{*}(T)$ up to algebraic equivalence: $T \sim V_{\Sigma}$.

(a2) $T$ is a nonunitary isometry $\left(T^{*} T=I \neq T T^{*}\right)$, in which case $T \sim S$.

(a3) $T$ is a nonunitary coisometry $\left(T^{*} T \neq I=T T^{*}\right)$, in which case $T \sim S^{*}$.

(a4) $T$ is neither isometric nor coisometric, in which case $T \sim S \oplus S^{*}$.

(b) If $T$ is a p.p.i. with finitely many nilpotent blocks of orders $k_{1}, \ldots, k_{n}$, then $P_{0}=I-\sum_{i=1}^{n} P_{k_{i}}$ is a projection in $C^{*}(T)$ and $T_{1}=P_{0} T \mid P_{0}$ is determined up to algebraic equivalence by (a). So $T \sim \bigoplus \sum_{i=1}^{n} N_{k_{i}} \oplus T_{1}$.

(c) If $T$ is a p.p.i. with nilpotent blocks of orders $k_{1}<k_{2}<k_{3}<\cdots$, then $T \sim$ $\bigoplus \sum_{i=1}^{\infty} N_{k_{i}}$.

Proof. If $T$ is a p.p.i. in a $C^{*}$ algebra $A$, represent $A$ on a Hilbert space and apply the theorem of [5]. Most of this proposition is straightforward, based on the simple fact that for any representation $\pi$ of $C^{*}(T)$, one has $T \sim T \oplus \pi(T)$.

Case (a1) follows from the spectral theorem. Since there is a $*$ homomorphism of $C^{*}(S)$ onto $C\left(S^{1}\right)$ taking $S$ to the identity function $z$, one has $S \sim S^{(\alpha)} \oplus V$ for any $\alpha \geq 1$ and any unitary $V$. Case (a3) is similar. Finally, the same remarks show that $S \oplus S^{*} \sim S^{(\alpha)} \oplus S^{*(\beta)} \oplus V$ for any $\alpha \geq 1, \beta \geq 1$ and any unitary $V$. The projections $P_{k}$ onto the nilpotent part of order $k$ belong to $C^{*}(T)$. Thus if there are only finitely many $P_{k_{i}}$ 's which are not zero, one can define $P_{0}=I-\sum_{i=1}^{n} P_{k_{i}}$. This pulls out a direct summand $T_{1}$ which is analyzed by part (a).

Given an infinite sequence $k_{1}<k_{2}<k_{3}<\cdots$, consider the p.p.i. $W=\bigoplus \sum_{i=1}^{\infty} N_{k_{i}}$ acting on $\mathcal{H}=\bigoplus \sum_{i=1}^{\infty} H_{i}$. Let $J$ be the ideal $C^{*}(W) \cap K$, where $K$ is the compact operators. Clearly $J$ contains the projection $P_{k_{i}}$ onto $\mathcal{H}_{i}$, and thus contains all of $B\left(\mathcal{H}_{i}\right) \cong M_{k_{i}}(\mathbf{C})$ for each $i \geq 1$. It follows that $J$ is the $c_{0}$ direct sum $\bigoplus \sum_{i=1}^{\infty} B\left(H_{i}\right)$. The quotient $C^{*}(W) / J$ is isomorphic to $C^{*}(\mathfrak{w})$ where $\mathfrak{w}$ is the image of $W$ in the Calkin algebra. Because $P_{k}$ is compact, $p_{k}(\mathfrak{w})=\pi P_{k}=0$ so $\mathfrak{w}$ is a p.p.i. with no nilpotent summands. Also,

$$
\mathfrak{w}^{*}=\pi W W^{*} \neq 1 \text { and } \mathfrak{w}^{*} \mathfrak{w}=\pi W^{*} W \neq 1,
$$

so $\mathfrak{w}$ is neither isometric nor coisometric. By (a), $\mathfrak{w} \sim S \oplus S^{*}$. Thus by (a) and (b),

$$
W \sim \bigoplus \sum_{i=1} N_{k_{i}}^{\left(\alpha_{i}\right)} \oplus S^{(\alpha)} \oplus S^{*(\beta)} \oplus V
$$

for any $\alpha_{i} \geq 1$, any $\alpha, \beta$ and any unitary $V$.

Theorem 2. Every p.p.i. $\mathrm{t}$ in the Calkin algebra lifts to a p.p.i. in $B(\mathcal{H})$. These p.p.i.'s are classified up to unitary equivalence in the Calkin algebra as follows.

(a1) If $\mathrm{t}$ is unitary and $\sigma(t)=\Sigma$ is a proper subset of $S^{1}$, then $\mathrm{t} \cong \pi\left(V_{\Sigma}^{(\infty)}\right)$. 
(a2) If $\mathbf{t}$ is unitary and $\sigma(\mathfrak{t})=S^{1}$, and ind $\mathbf{t}=n$, then $\mathfrak{t} \cong \pi\left(S^{(-n)}\right)$ if $n<0, \mathfrak{t} \cong$ $\pi\left(V_{S_{1}}\right)$ if $n=0$, and $\mathrm{t} \cong \pi\left(S^{*(n)}\right)$ if $n>0$.

(b) If $\mathrm{t}$ is isometric but not unitary, $\mathrm{t} \cong \pi\left(S^{(\infty)}\right)$.

(c) If $\mathrm{t}$ is coisometric but not unitary, $\mathrm{t} \cong \pi\left(S^{*(\infty)}\right)$.

(d) If $\mathrm{t}$ has no nilpotent part and is neither isometric nor coisometric, then $\mathrm{t} \cong$ $\pi\left(S^{(\infty)} \oplus S^{*(\infty)}\right)$.

(e) If $\mathrm{t}$ has finitely many nilpotent parts $k_{1}, \ldots, k_{n}$ with corresponding projections $\mathfrak{p}_{1}, \ldots, \mathfrak{p}_{n}$, then

$(\mathrm{e} 1-4)$ if $\mathfrak{p}_{0}=1-\sum_{i=1}^{n} \mathfrak{p}_{i}$ is not zero, then $\mathbf{t} \cong \pi\left(\bigoplus \sum_{i=1}^{n} N_{k_{i}}^{(\infty)} \oplus T_{1}\right)$ where $T_{1}$ is determined by $(\mathrm{a})-(\mathrm{d})$,

(e5) if $\mathfrak{p}=0$, then there is an integer $d$ with $0 \leq d<$ g.c.d. $\left\{k_{1}, \ldots, k_{n}\right\}$ such that $\mathfrak{t} \cong$ $\pi\left(\oplus \sum_{i=1}^{n} N_{k_{i}}^{(\infty)} \oplus O_{d}\right)$ where $O_{d}=N_{1}^{(d)}$ is the zero operator on a d-dimensional space.

(f) If $\mathrm{t}$ has nilpotent parts for the infinite sequence $k_{1}<k_{2}<k_{3}<\cdots$, then $\mathrm{t} \cong$ $\pi\left(\bigoplus \sum_{i=1}^{\infty} N_{k_{i}}^{(\infty)}\right)$.

Proof. Cases (a1) and (a2) follow from early results of [2]. Cases (b) and (c) are well known [4]. This is also immediate from the fact that $\operatorname{Ext}\left(C^{*}(S)\right)=0$. For case (d), we have $\mathrm{t} \sim S \oplus S^{*} \sim \pi\left(S^{(\infty)} \oplus S^{*(\infty)}\right)$. It suffices to show that $\operatorname{Ext}\left(C^{*}\left(S \oplus S^{*}\right)\right)=$ 0 . Let $T=S \oplus S^{*}$, and let $J$ be the ideal in $C^{*}(T)$ generated by $I-T^{*} T$. It is easy to see that $J$ is isomorphic to the compact operators $\mathcal{K}$, and that $C^{*}(T) / J$ is isomorphic to $C^{*}(S)$. Thus

$$
0 \rightarrow \mathcal{K} \rightarrow C^{*}(T) \rightarrow C^{*}(S) \rightarrow 0
$$

is exact. But $\operatorname{Ext} \mathcal{K}=0=\operatorname{Ext} C^{*}(S)$, and hence $\operatorname{Ext} C^{*}(T)=0$. So $\mathbf{t}$ is unitarily equivalent to $\pi\left(S^{(\infty)} \oplus S^{*(\infty)}\right)$.

In case (e5), $C^{*}(\mathrm{t})$ is isomorphic to $\bigoplus \sum_{i=1}^{n} M_{k_{i}}(\mathbf{C})$, and thus $\operatorname{Ext} C^{*}(\mathrm{t})$ is isomorphic to $\mathbf{Z} / p \mathbf{Z}$ where $p=$ g.c.d. $\left\{k_{1}, \ldots, k_{n}\right\}$ and $\operatorname{Ext}_{w} C^{*}(\mathbf{t})=0$ [7]. It follows readily that if $d$ is the integer with $0 \leq d<p$ such that $d+p \mathbf{Z}$ is the element of $\operatorname{Ext} C^{*}(\mathbf{t})$ determined by $\mathfrak{t}$, then $t \cong \pi\left(\bigoplus \sum_{i=1}^{n} N_{k_{i}}^{(\infty)} \oplus \mathcal{O}_{d}\right)$, and $\mathfrak{t} \cong{ }_{w} \pi\left(\bigoplus \sum_{i=1}^{n} N_{k_{i}}^{(\infty)}\right)$.

When $\mathfrak{p}_{0} \neq 0$, let $\mathfrak{t}=\mathfrak{t}_{0} \oplus \mathfrak{t}_{1}$ where $p_{0}=0 \oplus 1$. The above analysis shows that

$$
\mathrm{t} \cong \pi\left(\bigoplus \sum_{i=1}^{n} N_{k_{i}}^{(\infty)} \oplus O_{d} \oplus T_{1}\right) \cong \pi\left(\bigoplus \sum_{i=1}^{n} N_{k_{i}}^{(\infty)}\right) \oplus \pi\left(O_{d} \oplus T_{1}\right) .
$$

The latter term has no nilpotent part, so it is analyzed by parts (a) and (b).

Finally, if $\mathrm{t}$ has nilpotent parts for the infinite sequence $k_{1}<k_{2}<k_{3}<\cdots$, we refer back to the analysis of Proposition 1 to obtain an ideal $J$ isomorphic to $\bigoplus \sum_{i=1}^{\infty} M_{k_{i}}(\mathbf{C})$ in $C^{*}(\mathbf{t})$ so that

$$
0 \rightarrow \mathrm{J} \rightarrow C^{*}(\mathrm{t}) \rightarrow C^{*}\left(S \oplus S^{*}\right) \rightarrow 0 .
$$

By [7], Ext $J=0$. Since $\operatorname{Ext} C^{*}\left(S \oplus S^{*}\right)=0$, it follows that $\operatorname{Ext} C^{*}(\mathfrak{t})=0$. Thus $\mathfrak{t}$ is unitarily equivalent to $\pi\left(\bigoplus \sum_{i=1}^{\infty} N_{k_{i}}^{(\infty)}\right)$.

REMARKs. (1) One of the most surprising facts, noted first in [5], is that $\bigoplus \sum_{k=1}^{\infty} N_{k}$ is approximately unitarily equivalent to $S^{(\infty)} \oplus S^{*(\infty)}$. This is now "explained" by noting that, in the Calkin algebra, both have nonisometric, noncoisometric images without nilpotent parts, and hence the images are algebraically equivalent to $S \oplus S^{*}$. This can be represented in the Calkin algebra in only one way. 
(2) Except in case (e5) in which $C^{*}(\mathrm{t})$ is finite dimensional, weak and strong equivalence coincide for p.p.i.'s.

(3) The classification of p.p.i.'s in $B(\not{H})$ up to approximate unitary equivalence is carried out in [6]. These results are also immediate from Theorem 2 . In fact, it would be sufficient to show every p.p.i. in the Calkin algebra lifts to a p.p.i. without classifying them as one goes along. But the nature of our method forces us to do it all.

\section{REFERENCES}

1. W. B. Arveson, Notes on extensions of $C^{*}$ algebras, Duke Math. J. 44 (1977), 329-355.

2. L. G. Brown, R. G. Douglas and P. A. Fillmore, Unitary equivalence modulo the compact operators and extensions of $C^{*}$ algebras, Lecture Notes in Math., Vol. 345, Springer-Verlag, Berlin and New York, 1973.

3. M. D. Choi and E. G. Effros, The completely positive lifting problem, Ann. of Math. (2) 104 (1976), 265-324.

4. P. R. Halmos, Limits of shifts, Acta Sci. Math. (Szeged) 34 (1973), 131-139.

5. P. R. Halmos and L. J. Wallen, Powers of partial isometries, J. Math. Mech. 19 (1970), 657-663.

6. D. A. Herrero, Unitary orbits of power partial isometries and approximation by block diagonal nilpotents, Topics in Modern Operator Theory, Birkhäuser, Basel, 1981, pp. 171-210.

7. M. Pimsner and S. Popa, On the Ext-group of an AF-algebra, Rev. Roumaine Math. Pures Appl. 23 (1978), 251-267.

8. D. Voiculescu, A non-commutative Weyl-von Neumann theorem, Rev. Roumaine Math. Pures Appl. 21 (1976), 97-113.

Department of Pure Mathematics, University of Waterloo, Waterloo, Ontario N2L 3G1, CANADA 